
\title{
28 Research Square \\ Automated custom-fitted 3D-printed masks using free software and face scans
}

\author{
Edward J Sammut \\ University of Malta \\ Yin Chien Yeap \\ University of Malta \\ Jonathan Quan-Hui Yeap \\ University of Malta \\ Gustavo Mendonca \\ University of Michigan \\ Arthur Rodriguez Gonzalez Cortes ( $\sim$ arthur.nogueira@um.edu.mt ) \\ University of Malta https://orcid.org/0000-0001-6591-7256
}

\section{Technical Note}

Keywords: COVID-19, face mask, 3D printing, face scanning, computer-aided design, computer-aided manufacturing

Posted Date: April 30th, 2020

DOl: https://doi.org/10.21203/rs.3.rs-24633/v1

License: (c) (i) This work is licensed under a Creative Commons Attribution 4.0 International License. Read Full License 


\section{Abstract}

In light of the COVID-19 pandemic a global shortage of personal protective equipment (PPE) made necessary the development of techniques to rapidly design and adapt filtering masks for local manufacturing using 3D printed methods to help protect healthcare workers. Several Standard Tessellation Language (STL) files of 3D-printed masks have become available in the internet and some models have got approval from regulatory organisations such as the Food and Drug Administration (FDA). This technical report aimed at describing a straightforward methodology for custom-fitting 3Dprinted filtering masks to 3D face scans using free software. This article presents modifications of an open-source add-on program (My Face Mask, Wasp, Massa Lombarda, Italy) developed for a free computer aided-design (CAD) software (Blender 2.82, Amsterdam, Netherlands) to design a 3D-printed face mask with polylactic acid (PLA) filament, for P3 filters (99.95\% filtration of airborne particles) from face scan STL files. Preliminary results demonstrated that a dental clinician was able to digitally design and 3D-print a mask that provided satisfactory comfort and adaptation. The use of online application for a face scan and an open source software can be used to custom fit a 3D-printed mask to on individuals face.

\section{Introduction}

The COVID-19 pandemic has resulted in a global shortage of personal protective equipment (PPE). This stimulated the emergence of several designs of filtering masks for local manufacture using threedimensionally (3D) printed methods. ${ }^{1}$ The initial perceived advantage was about creating a supply in a situation of shortage from commercial manufacturers. However, 3D printing technologies offer the advantage of customisation to ensure a perfect fit for the end user. This may be helpful in alleviating two key issues when utilizing filtering masks. In the first instance, the quality of fit reduces or eliminates leakage of air around the mask, enhancing the safety of the user. Secondly, a well-fitting mask does not place undue pressure on the user's face resulting in more comfortable wear for prolonged periods, applicable to situations where extended use without removal of the mask is indicated. ${ }^{2}$

"My Face Mask" was an open source project by WASP (CSP S.r.l., Italy) published on their company website in March. ${ }^{3}$ Their method utilised photogrammetry to obtain a 3D mesh of the user's face. This mesh was used to adapt a pre-existing design using open-source software (Blender, Blender Institute, Netherlands), and a proprietary plug-in for Blender 2.82 released by WASP. The mask was designed for printing on fused filament deposition printers. "My Face Mask" utilizes a sheet of filter material that was held inside the mask using a retaining ring.

Healthcare worker infection with COVID-19 has been found to be related to several factors, with availability and quality of PPE found to be an important factor. ${ }^{1}$ In this COVID-19 pandemic hospitals which implemented rigorous PPE protocols including face masks with P3R filters (i.e. filters offering efficiency of $99.95 \%$ for airborne particles) apparently were able to avoid infections of staff, even in severely affected countries. ${ }^{4,5}$ 
The aim of this article is to present a straightforward method for digitally designing a 3D-printed customised mask, with improvements and modifications proposed on a plug-in (My Face Mask) of a free software. First, the method of facial scan acquisition was changed to use a mobile phone handset to make image acquisition faster and more user friendly. Second, the front of the mask was redesigned with a thread fitting $40 \mathrm{~mm} \times 1 / 7$ " (EN 148-1:1999) to accept widely used P3R filters. Thirdly, materials were selected for production based on desirable properties and sample prints were produced for a volunteer.

\section{Materials And Methods}

This technical report was conducted on one of the authors (ARGC) who volunteered to receive an individualised mask used to illustrate this technical report. The volunteer signed an informed consent to confirm his will to participate in this study. This study followed the Helsinque Declaration guidelines.

Before using any software, the "My Face Mask" plug-in was downloaded from the WASP website, installed in a computer-aided design software (Blender v. 2.82, Blender Institute, Amsterdam, Netherlands) and enabled. The plug-in basic mask design was edited to include a thread fitting $40 \mathrm{~mm} \times 1 / 7$ " (EN 1481:1999) on the front plate. The modified design tool was uploaded to a software development online platform (GitHub, San Francisco, CA, USA) and named "MyFaceMask Twist". A facial scan of a volunteer was obtained using the mobile phone app Bellus 3D FaceApp (Bellus3D, Campbell, CA, USA) and exported as STL files.

In Blender software, the plugin was initiated, and the face model was opened (the plugin can accept STL and OBJ files). The plugin allowed a re-mesh procedure to fill any gaps in the 3D model. The model was rotated to position the face forward and this was fine-tuned to ensure the nose was pointing forward using the vertical viewport. The model was moved so that the tip of the nose was on the axis of the horizontal plane. Next, the "Define area" function was used to draw to the border of where the edge of the mask should meet the face (Fig. 1). Once the area for contact of the mask with the face was defined, the "Adapt mask" button was clicked resulting in a displayed mask shell fitted to the face model (Fig. 2). Mask placement could be adjusted by selecting the face model and repositioning it. The plug-in allows for symmetric borders to mirror one edge over the opposite side of the face as well as adjustment for nose pressure, mask thickness and offset. The next step was to position the front plate, in lateral view, using the "Align filter on" button (Fig. 3). Some changes caused the mesh to re-generate itself, occasionally with defects, therefore, if defects were detected a different adjustment point was selected. Figure $2 \mathrm{C}$ illustrates resulting mesh errors caused by a collision between the filter plate and the face model, which could be then avoided by readjusting the filter position. Finally, elastic strap holes were positioned using the "show holes" button (Fig. 4) and resized using the Scale tool. Once the mask design was satisfactory, the "Align filter off" button was selected to return the model to a solid (Fig. 5), which could then be prepared and exported as STL file to be 3D-printed (Fig. 6), following previous methodology. ${ }^{6}$ The resulting STL was then analysed in a 3D software (Simplify3D software, Simplify3D, Cincinnati, USA) before undergoing 3D-printing tests. 


\section{Results}

The resulting STL analysis confirmed that the file was ready for 3D printing with no failures in the mesh composition. The process was set up for slicing using the following settings: Nozzle Diameter $0.4 \mathrm{~mm}$; Extrusion Multiplier 1.02; Extrusion Width $0.4 \mathrm{~mm}$; Retraction $1.6 \mathrm{~mm}$; Layer height $0.2 \mathrm{~mm}$; Solid layers (top/bottom) 3; Shells 2; Outline direction inside-outside. Random start points for all perimeters enabled, Extruder temperature set at 230खC with heated print bed temperature at 110冈C and Print speed set at $25 \mathrm{~mm} / \mathrm{sec}$.

After slicing, the resultant instructions were saved to SD card media and printed in a Flashforge Creator Pro 3D printer (Flashforge, Jinhua City, China) using thermoPlastic copolyester (Arnitel 2045 filament, Koninklijke DSM N.V., Geleen, Netherlands). Print was allowed to cool fully before removing from the print bed. A threaded filter adapter (Sr 280-3, Sundstrom safety AB, Lagan, Sweden) was fitted and a P3 R filter from the same manufacturer (SR510 P3R) conforming to EN 143:2000 P3 R > 99.997\% together with the appropriate pre-filter assembled.

The total time required for digitally designing the mask is one hour approximately, while the time required for 3D-printing all mask components was 12 hours, considering the facial dimensions of the wearer. The resulting 3D-printed mask (Fig. 7) could be used by the volunteer, providing satisfactory adaptation and comfort.

\section{Discussion}

This paper aimed at describing a feasible technique for digitally customising 3D-printed face masks to individual face scans, which offers some important advantages. Firstly, such methodology customisable using free software thereby lowering the barrier to individual use of the design and offering a relatively cheap solution for public health sectors. Secondly, when placed filter-side down on the print-bed, it was possible to print the mask without supporting structures as each layer would have sufficient overlap from the previous layer to support it (Fig. 4). This feature results in savings of time when printing as well as avoiding material wastage.

Therefore, the main advantages of the proposed methodology are: 1) customisation for perfect fit resulting in avoidance of air leakage and extended-use comfort, 2) local fabrication eliminating issues with supply chain disruption during crisis, 3 ) reusable item of equipment lowering long term costs of PPE (It is also possible to scribe text on the model, for mask user identification), and 4) documented high levels of particle filtration by using a tried and tested filter system. The main limitations include requirement for technology application (Apple Iphone and computer able to run Blender, 3D printer with sufficient features to print more advanced filaments, human resources trained to use all of these items), an extensive printing time of around 5 hours per mask, supply of 3D printing filament and P3R filters and, high initial costs per mask. 
In searching for a filter material solution, it was clear that a P3 standard of filtration was desirable considering the small dimension of the coronavirus and the need to protect healthcare workers to prevent failure of healthcare systems in the face of the pandemic. ${ }^{2}$ Therefore, the front of the mask was redesigned with a thread fitting $40 \mathrm{~mm} \times 1 / 7$ " (EN 148-1:1999), to which the P3R filters of several manufacturers can be fitted. The P3 standard provides a high level of protection filtering more than 99.97\% of airborne particles and protects against solid and liquid aerosols. P3 filters have an Occupational Exposure Limit (OEL) rating of 50x and an Assumed Protection Factor (APF) of 20x.

A P3R filter is reusable and may be used for several shifts. Some manufacturers suggest use of a P3R filter can be extended to several months. Filters can be disinfected with alcohol in between shifts, and in the selected system, a disposable prefilter eliminates most of the particle load, sparing the main filter. These features increase the system's economy. In addition, the manufacturer instructions for use for the SR-510 P3R filter allow an operating temperature of 55खC for this filter. Meanwhile, data from a study looking at inactivation of a similar virus (SARS-Cov) revealed that in the absence of protein contamination, the virus could be inactivated by a temperature of $56 \otimes C{ }^{7}$ It is not unreasonable, within the headroom allowed by manufacturers, that disinfection in a warm-air oven at 56खC would inactivate the viral load and preserve the filter's properties. Thermal sensitivity testing of the current pathogen is needed, and manufacturer testing of their filters at $60 \otimes \mathrm{C}$ or higher is also needed, to permit more certainty in reprocessing of filters for reuse.

The material used herein was a thermoplastic copolyester, which is produced from $50 \%$ bio-based feedstock and is resistant to high temperatures due to long term thermo-oxidative stability as well as good UV resistance. Such material is highly elastic with a Shore D of 24 and passes ISO 10993-10 irritation/intracutaneous reactivity testing, ISO 10993-5 cytotoxicity testing and USP class VI for medical implants. Critically for this application, it has a high inter-layer adhesion due to its slower crystallization behaviour. The manufacturer suggests it for wearable items such as smartwatch bands as well as mouthguards. Our initial 3D printing tests of the mask design resulted in failed prints due to various problems, which were solved by adopting the final printing settings described above. Such settings are applicable to the specific model of 3D printer used, while others may require different settings.

Among the limitations of the present methodology are: the complex photogrammetry techniques used to capture the 3D image of the face and the use of a thin filter material in the filter holder at the front of the mask. To overcome the first limitation, various facial scanning methods were considered, including using intraoral dental scanners to scan the facial skin, using desktop model scanners to scan casts of the face and using various mobile phone methods. The choice of Bellus3D was based on the favourable ratio between the quality of the facial scan versus the technique's simplicity and speed, accepting that it is limited to functioning only on recent Apple Iphone ${ }^{\text {TM }}$ (Apple Inc, Cupertino, CA) mobile phone models. Since the method is quick, one unit of hardware can be used to scan several faces per hour, maintaining the concept of ease of accessibility for our concept. Furthermore, the technical report design of this article aimed only at showing the potential of an automated software tool tool to customise 3D-printed masks to face scans. Therefore, future prospective laboratory and clinical would be recommended to address 
qualitatetive and quantitative fit results, as well as the long-term stability and airborne particle protection levels. Finally, despite the automated use of the plug-in presented herein, basic CAD knowledge is still required in this method. On the other hand, software straightforward tools and plug-ins are currently being developed to simplify digital design procedures, especially considering the current focus on solutions to prevent COVID-19 transmission. Even if some level of supply of FFP3 masks is established, these need to be available in a variety of sizes and properly fit-tested for each healthcare worker.

\section{Conclusion}

The present findings suggest that the method for fabrication of custom fitted facial masks with P3R filters outlined herein may be a viable and effective alternative to the commercial supply.

\section{Declarations}

Ethics approval and consent to participate: Following the procedure recommended by the local ethics committee (CEP), the volunteer (who is an author) of the case presented herein to illustrate the technique agreed with participating in the report by signing an informed consent.

Consent for publication: Not applicable

Availability of data and material: Treatment and software data of the presented case is available with the authors.

Competing interests: None.

Funding: No funding was available for this study:

Authors' contributions Edward Sammut And Chien Yeap performed the digital design in the software. Jonathan Yeap performed 3D-printing procedures. Gustavo Mendonça performed facial scanning procedures. Arthur C. performed software STL superimposition measurements and text writing. All authors reviewed the text scientifically.

Acknowledgements: None.

\section{References}

1. Adhikari SP, Meng S, Wu YJ, Mao YP, Ye RX, Wang QZ, Sun C, Sylvia S, Rozelle S, Raat H, Zhou H. Epidemiology, causes, clinical manifestation and diagnosis, prevention and control of coronavirus disease (COVID-19) during the early outbreak period: a scoping review. Infect Dis Poverty. 2020;9:29.

2. Lei Z, Yang J, Zhuang Z. Headform and N95 filtering facepiece respirator interaction: Contact pressure simulation and validation. J Occup Environ Hyg. 2012;9:46-58.

3. WASP c/o CSP S.r.I 
WASP c/o CSP S.r.I. Inc. (2020) MY FACE MASK: 3D printed face Mask with a replaceable filter. https://www.3dwasp.com/en/3d-printed-mask-from-3d-scanning/ Accessed March 21, 2020.

4. Makowski K, Okrasa M. Application of 3D scanning and 3D printing for designing and fabricating customized half-mask facepieces: A pilot study. Work. 2019;63:125-35.

5. Pacitto A, Amato F, Salmatonidis A, Moreno T, Alastuey A, Reche C, Buonanno G, Benito C, Querol X. Effectiveness of commercial face masks to reduce personal PM exposure. Sci Total Environ. 2019;650:1582-90.

6. Spoerk M, Gonzalez-Gutierrez J, Sapkota J, Schuschnigg S, Holzer C. Effect of the printing bed temperature on the adhesion of parts produced by fused filament fabrication. Plast Rubber Compos. 2018;47:17-24.

7. Rabenau HF, Cinatl J, Morgenstern B, Bauer G, Preiser W, Doerr HW. Stability and inactivation of SARS coronavirus. Med Microbiol Immunol. 2005;194:1-6.

\section{Figures}




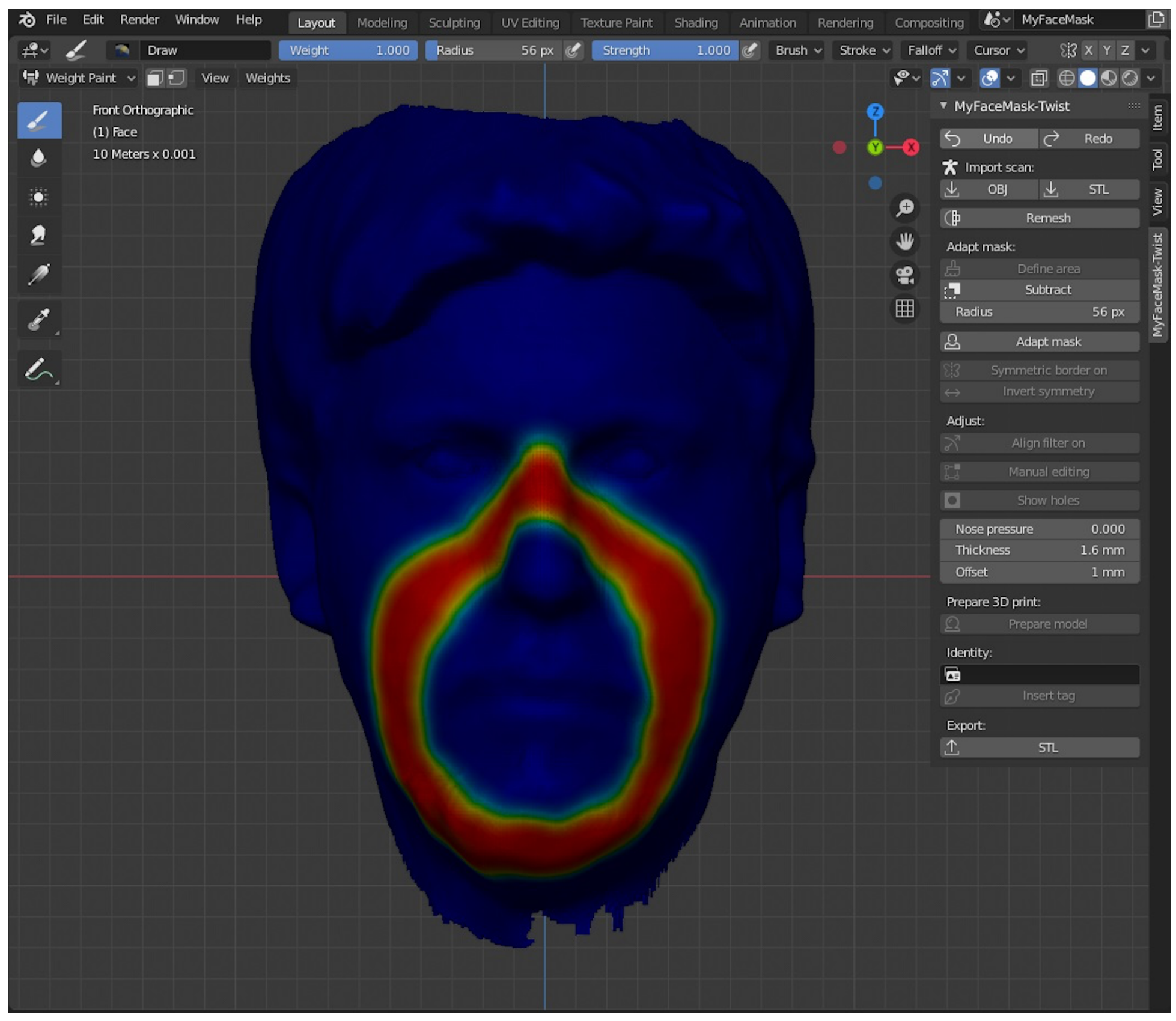

\section{Figure 1}

Face model (STL file) rotated to position with face forward and define area function in plug-in used to draw mask border on the face. 


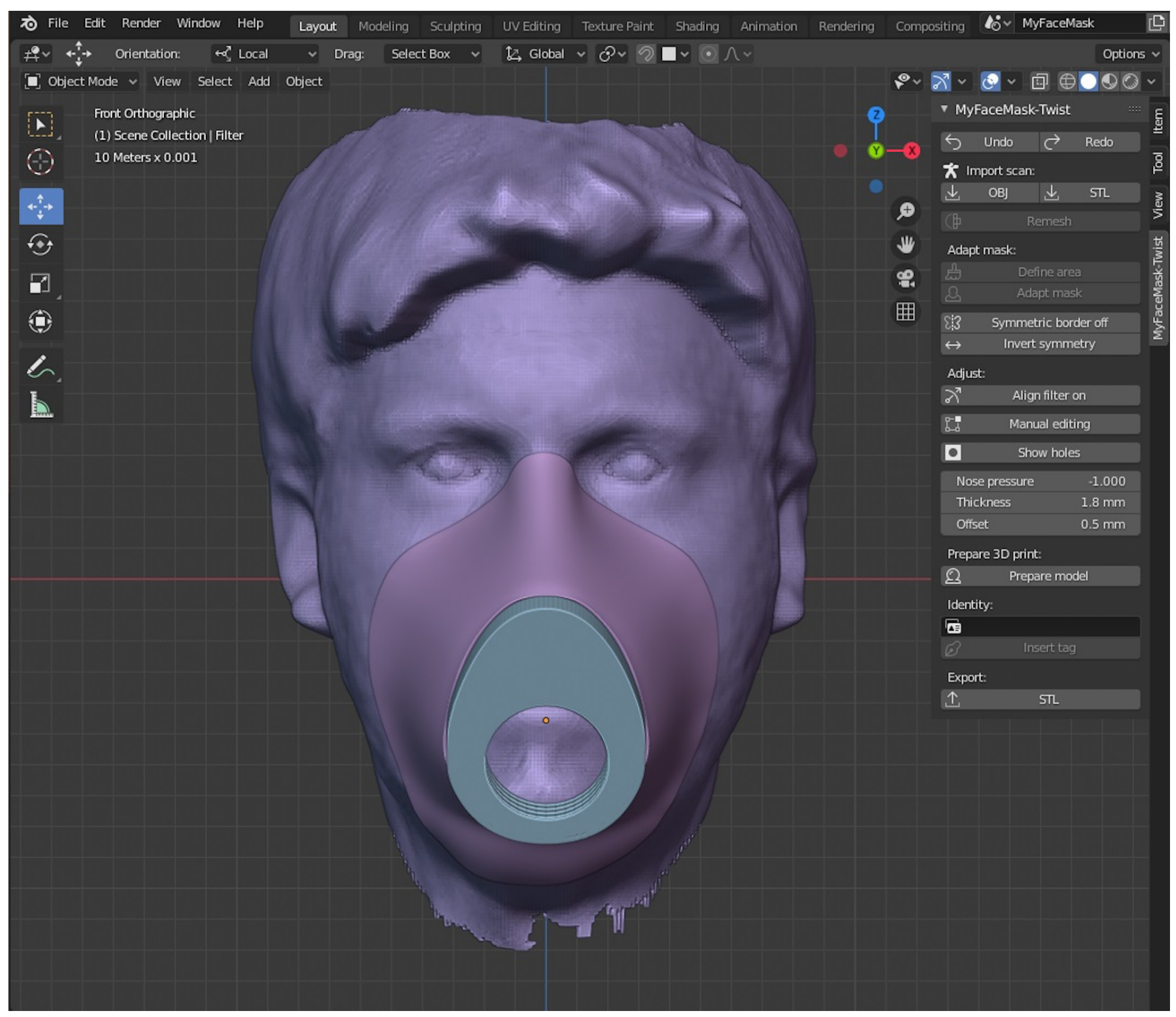

Figure 2

After "Adapt mask" function clicked, the mask shell was fitted to the face. 


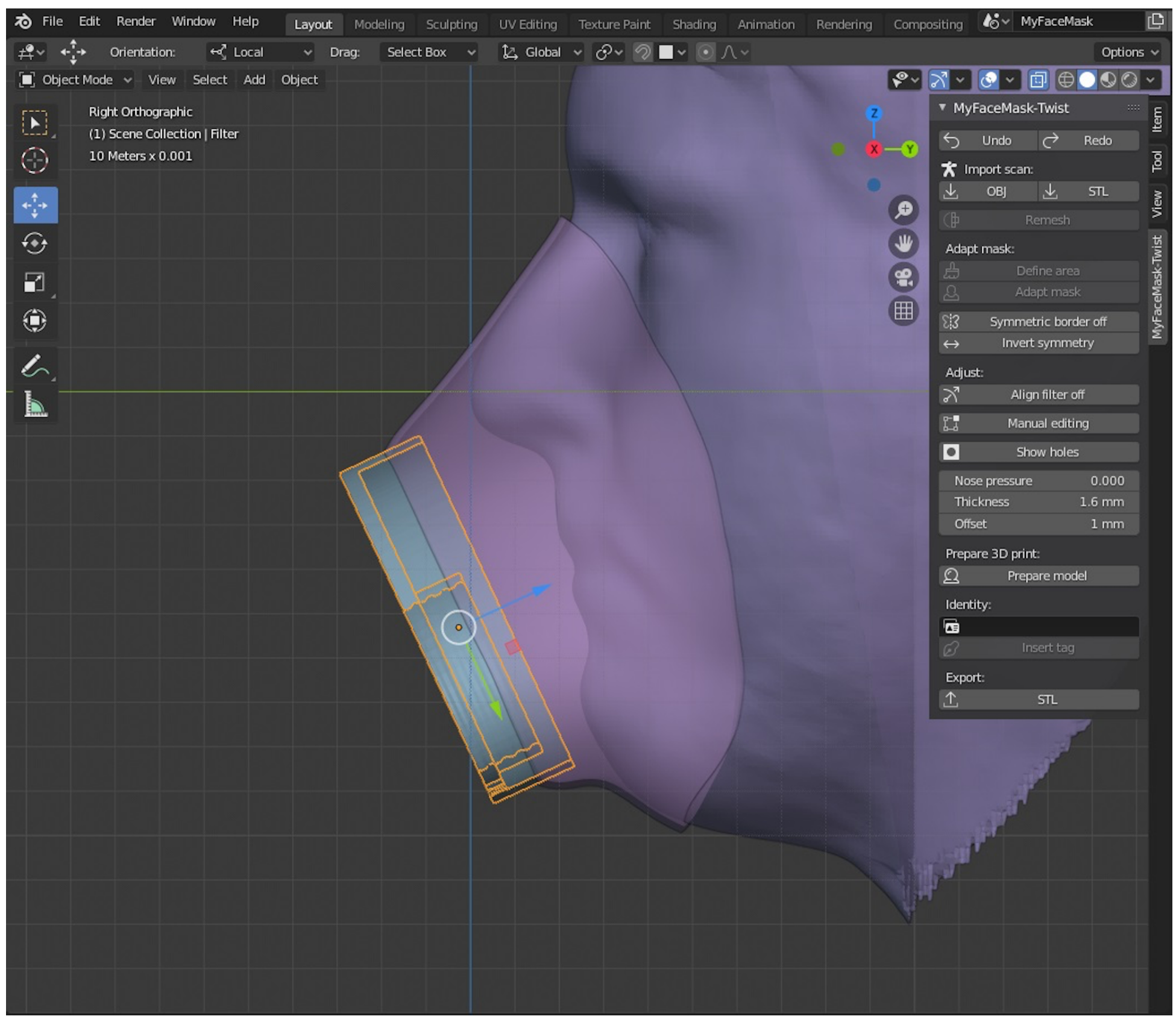

\section{Figure 3}

The position of the filter plate can be changed and refined using translate and rotate with the "Align Filter On" function. 


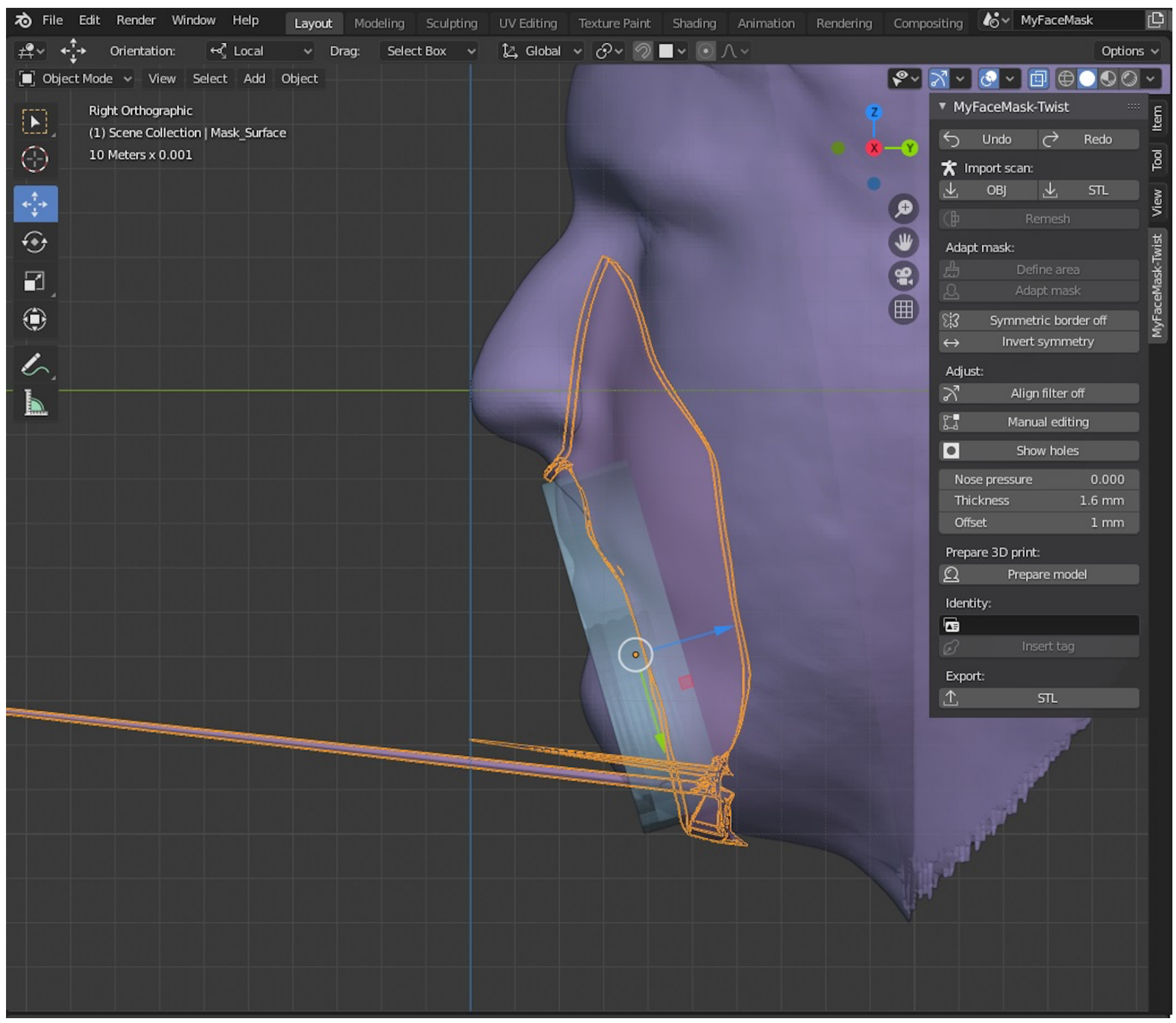

\section{Figure 4}

Incorrect positioning of the parts of the mask lead to mesh errors. In this case, the filter plate is colliding with the face model. 


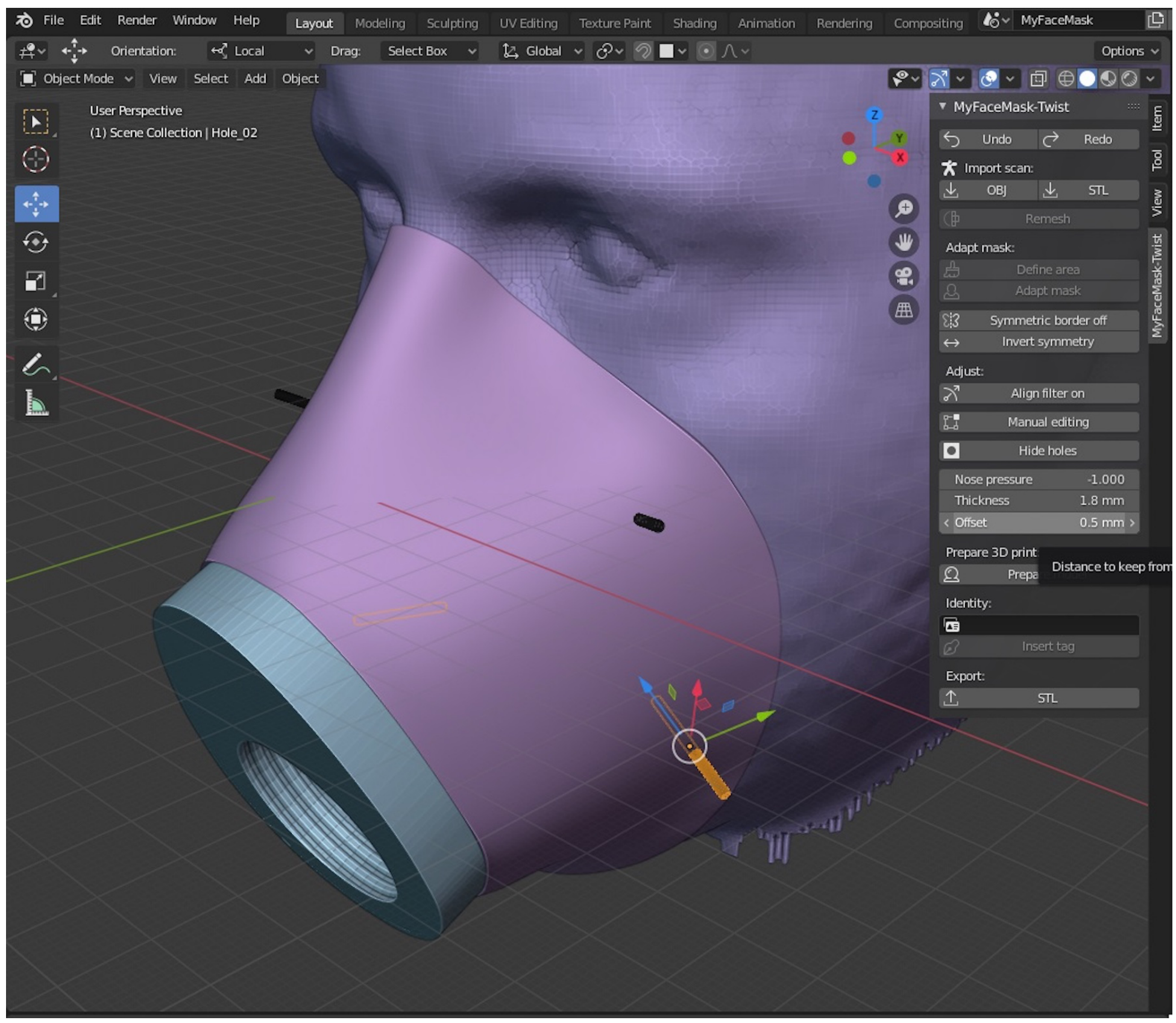

\section{Figure 5}

"Show Holes" function can be used to position the strap holes. Their dimensions can be altered in the Scale settings. 


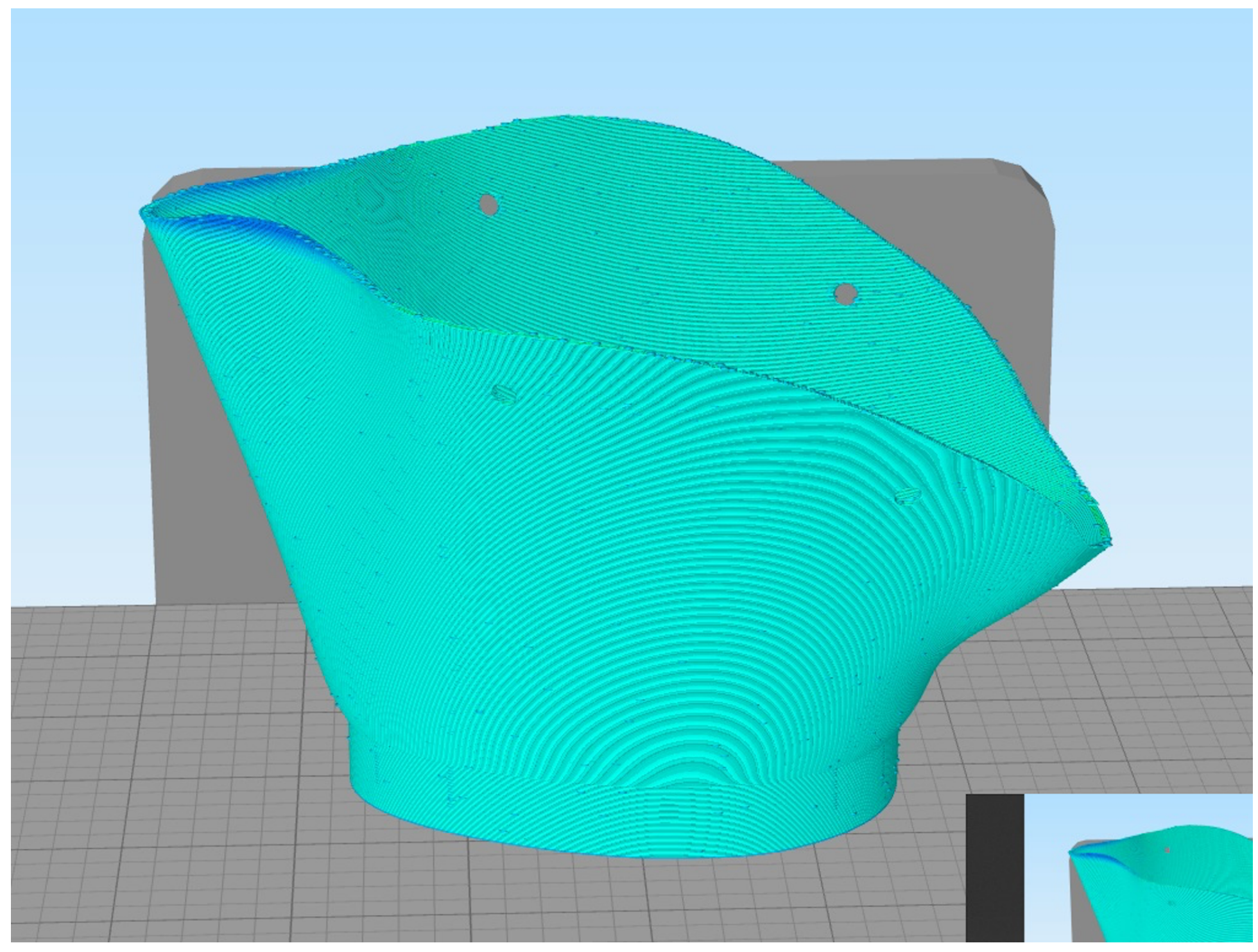

\section{Figure 6}

Sliced mesh in 3D printing software (Simplify 3D) showing print possible without support material 


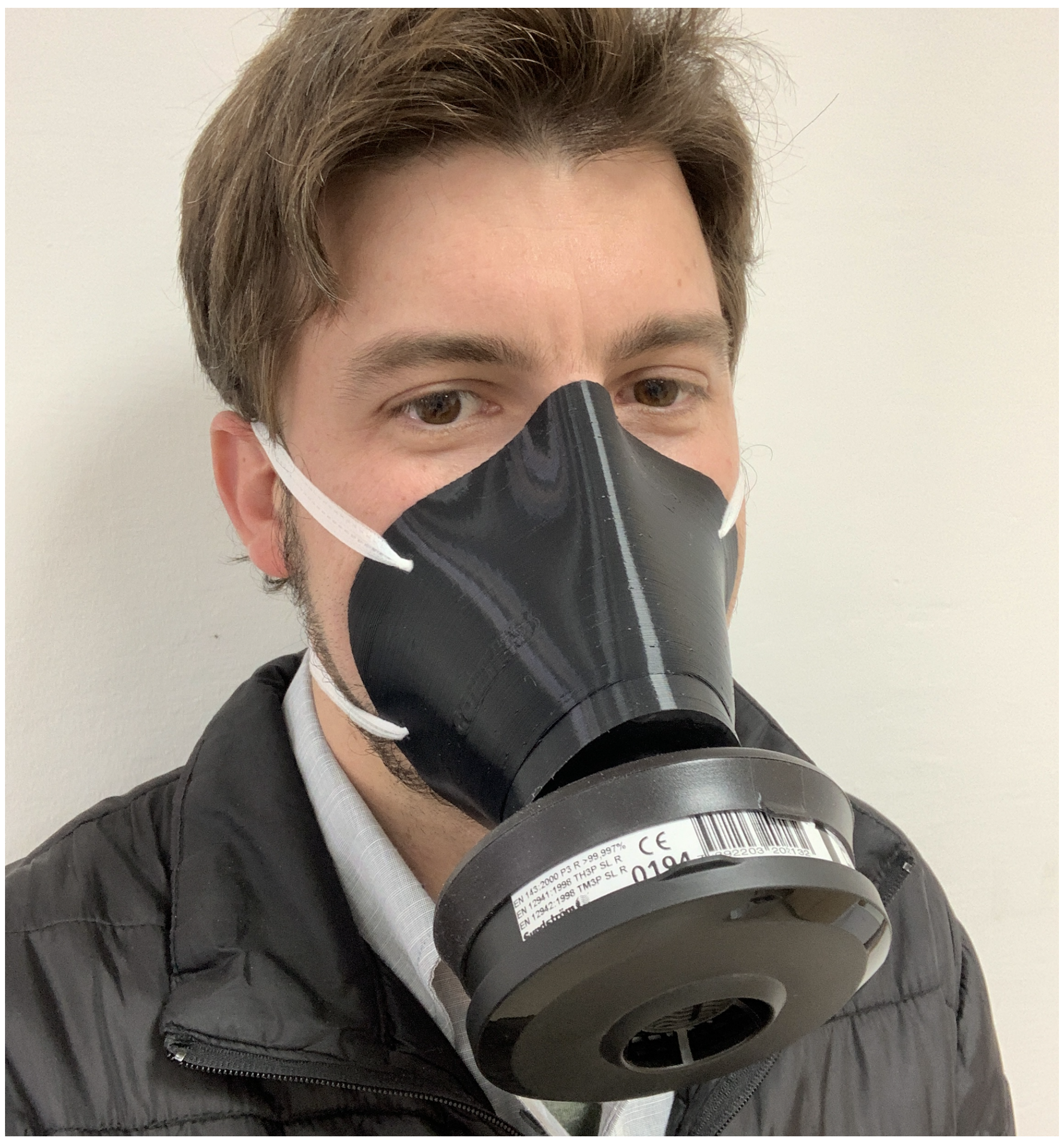

Figure 7

Final result of the 3D-printed mask. 